\title{
Downlink Capacity Analysis of Distributed Antenna Systems with Imperfect Channel State Information
}

\author{
Weiye $\mathrm{Xu}^{1,2}$, Min Lin ${ }^{1}$ \\ ${ }^{1}$ College of Communications Engineering, PLA University of Science and Technology, Nanjing, China, \\ ${ }^{2}$ School of Communication Engineering, Nanjing Institute of Technology, Nanjing, China \\ *Corresponding author: Weiye $\mathrm{Xu}$
}

Received December 20, 2015; revised March 18, 2016; accepted November 28, 2016; published January 31, 2017

\begin{abstract}
In this paper, considering that perfect channel state information (CSI) is hard to achieve in practice, the downlink capacity of distributed antenna systems (DAS) with imperfect CSI and multiple receive antennas is investigated over composite Rayleigh fading channel. According to the performance analysis, using the numerical calculation, the probability density function (PDF) of the effective output SNR is derived. With this PDF, accurate closed-form expressions of ergodic capacity and outage probability of DAS with imperfect CSI are, respectively, obtained, and they include the ones under perfect CSI as special cases. Besides, the outage capacity of DAS in the presence of imperfect CSI is also derived, and a Newton's method based practical iterative algorithm is proposed to find the accurate outage capacity. By utilizing the Gaussian distribution approximation, another approximate closed-form expression of outage capacity is also derived, and it may simplify the calculation of accurate outage capacity. These theoretical expressions can provide good performance evaluation for downlink DAS for both perfect and imperfect CSI. Simulation results verify the effectiveness of the theoretical analysis, and the system capacity can be improved by increasing the receive antennas, and decreasing the estimation error or path loss. Moreover, the system can tolerate the estimation error variance up to about 0.01 with a slight degradation in the capacity.
\end{abstract}

Keywords: Distributed antenna systems, imperfect CSI, ergodic capacity, outage capacity, path loss, multiple receive antennas. 


\section{Introduction}

Distributed antenna system (DAS), as a promising technique for next generation wireless mobile communications, has received much attention due to its power efficiency and capacity improvement over the traditional co-located antenna system (CAS) [1][2][3][4][5]. Different from the CAS, the remote antennas in DAS are separated geographically and connected to a central processing unit (CPU) via dedicated wires, fiber optics, or an exclusive radio frequency link. The antenna selection transmission with maximal-ratio combining (MRC) is an effective diversity scheme to increase the performance while significantly decreasing the hardware complexity and cost [6][7]. Therefore, effective combination of DAS and antenna selection technique will improve the system performance greatly with efficient hardware complexity and cost.

Channel capacity, as a measure indicator of the system performance, plays important role in communication systems. Based on different system models, the capacity performance of DAS has been extensively studied in [8]-[26] and references therein. The performance of downlink DAS over multicell Rayleigh and Nakagami fading channels are, respectively, analyzed in [8] and [9], where the closed-form expressions of ergodic capacity are derived for the corresponding fading channels, but the derived expression in [8] has minor error, and [9] needs iterative calculation. For this reason, an accurate closed-form expression of ergodic capacity of downlink DAS in Nakagami fading multicell environment is derived in [10]. The ergodic capacity of downlink DAS is analyzed in [11] by using different cooperation strategies, and an adaptive cooperation is presented to improve the capacity, but the influence of noise is neglected for analysis simplicity. In [12], the uplink ergodic sum capacity performance of multicell and multiuser DAS is studied under an interference-limited scenario. Considering single cell composite fading (including small- and large-scales fading), the capacity performance of DAS is studied in [13]-[19]. In [13], [14], [15] and [16], approximate expressions of the ergodic capacity are derived for downlink DAS over composite Rayleigh and Nakagami fading channel, respectively, but the derived capacity expressions are not accurate enough to reflect the actual values, and the analysis is limited in single receive antenna case. For this, tightly-approximate closed-form expressions of ergodic capacity for downlink DAS with multiple receive antennas are provided in [17], which are more accurate than the previous expressions. By high signal to noise ratio (SNR) analysis, an approximate expression of ergodic capacity of downlink DAS is derived in [18], which is close to the actual cell average ergodic capacity at high SNR. By assuming double-sided spatial correlation, an analytical lower bound of the ergodic capacity of uplink DAS in composite Rayleigh fading channel is provided in [19], but the capacity is accurate in the low and high SNR regimes only. Besides, the outage performance of DAS in fading channel has been also studied. For the downlink, an approximate outage probability is derived for DAS with single receive antenna over composite Rayleigh fading channels in [20]. For the uplink, the approximate outage probability is analyzed and derived for DAS in composite Rayleigh fading channels [21][22], in composite Nakagami fading channels [23], and in composite generalized-gamma fading channels [24], where the mobile terminal (MT) is equipped with one antenna only. In [25], a closed-form approximation of the outage probability for the DAS downlink and uplink is derived, and this approximation is tight only when the rate or the number of nodes and antennas of the system becomes large. The impact of MRC-based macro-diversity on the capacity of code division multiple access (CDMA) DAS is analyzed in [26], and the expressions of outage probability on the reverse link and the forward link are derived, 
respectively.

Although in all these studies, the capacity and outage performances are well analyzed, the performance analysis is based on perfect channel state information (CSI), whereas in practice, the perfect CSI is difficult to obtain due to channel estimation error. Moreover, most of the above references consider single receive antenna case for analysis convenience, so the derived capacity expressions lack generality and the system performance is also limited. Motivated by the reasons above, we will address the performance study of downlink DAS with transmit antenna selection and multiple receive antennas in the presence of imperfect CSI, where both small-scale Rayleigh fading and large-scale path loss are considered. The main contributions are summarized as follows:

1) The ergodic capacity of DAS in the presence of imperfect CSI is analyzed. According to the performance analysis under imperfect CSI, and using numerical calculations, the probability density function (PDF) of the effective output SNR of DAS is derived. With this result, accurate closed-form expressions of ergodic capacity and second moment of channel capacity are achieved, respectively. These expressions match the corresponding simulations very well. Thus, they can provide good theoretical evaluation for downlink DAS capacity under imperfect CSI.

2) The outage performance of DAS with imperfect CSI is presented, and an accurate closed-form expression of outage probability of DAS for a given outage capacity is derived. For a given outage probability, a practical iterative algorithm based on Newton's method is presented for obtaining the accurate outage capacity. Besides, by utilizing the Gaussian distribution approximation, an approximate closed-form expression of outage capacity of DAS is also derived, and it has the value close to accurate one, but no iteration is required. With these expressions, the outage performance of downlink DAS under imperfect CSI can be effectively evaluated, and correspondingly, the conventional requirement for Monte Carlo simulation will be avoided.

3) The derived theoretical expressions include the ones under perfect CSI and/or single receive case as special cases. Thus, they can also be used for evaluating the performance of downlink DAS under perfect CSI. For the given outage probability, it is shown that the solution of outage capacity does exist and is unique. Based on this, we propose to use Newton's method to obtain the solution due to its quadratic convergence rate.

The notations we use throughout this paper are as follows. Bold upper case and lower case letters denote matrices and column vectors, respectively. $E\{\cdot\}$ denotes the expectation. The superscripts $(\cdot)^{T}$ is used to stand for the transpose. $\mathbf{I}_{N}$ denotes the $N \times N$ identity matrix.

\section{System Model}

In this paper, we consider a DAS with $M$ remote antennas (RAs) and $N$ receive antennas in a single cell environment as shown in Fig. 1, the RAs are distributed in the cell and connected with the central base station (BS, also named as $R A_{1}$ ) via coaxial cables, fiber optics or radio links, where the $i$-th RA is denoted as $\mathrm{RA}_{i}$, and the MT is equipped with $N$ antennas. It is assumed to be independent and uniformly distributed in the cell as in [8] [18]. To improve the system performance, the antenna selection diversity scheme is employed for RAs. If $R A_{i}$ $(i=1, \ldots, M)$ is selected to transmit the signals, the received signals at MT can be expressed as

$$
\mathbf{y}_{i}=\sqrt{P_{t}} \mathbf{h}_{i} b+\mathbf{z}=\sqrt{P_{t}}\left[h_{1, i}, h_{2, i}, \ldots, h_{N, i}\right]^{T} b+\mathbf{z}
$$


where $\mathbf{y}_{i}=\left[y_{1, i}, y_{2, i}, \ldots, y_{N, i}\right]^{T}, y_{j, i}$ is the received signal from the $j$-th antenna at MT, $P_{t}$ is the transmit power. $h_{j, i}$ is the element of channel vector $\mathbf{h}_{i}$ and denotes the composite channel fading coefficient between $R A_{i}$ and the $j$-th antenna of MT. $b$ is the transmitted signal from $R A_{i}$ with unity energy. $\mathbf{z}$ is the noise vector, whose elements are independent, identically distributed (i.i.d) complex Gaussian random variables (r.v.s) with zero-mean and variance $\sigma_{z}^{2}$.

Due to the structural feature of DAS, $h_{j, i}$ can be modeled as $h_{j, i}=g_{j, i} \sqrt{L_{i}}$, where $g_{j, i}$ represents the small-scale fading between $R A_{i}$ and the $j$-th receive antenna of MT. For Rayleigh fading channel, $\left\{g_{j, i}\right\}$ are modeled as independent complex Gaussian r.v.s with zero-mean and unit-variance. $L_{i}$ denotes the pass loss between $R A_{i}$ and MT, and is expressed as $L_{i}=\left(d_{0} / d_{i}\right)^{\alpha_{i}}$, where $\alpha_{i}$ is the path loss exponent, $d_{0}$ is the reference distance and $d_{i}$ represents the distance from $R A_{i}$ to MT.

According to (1), when single $R A_{i}$ is selected, the output SNR after MRC can be obtained as

$$
\rho_{i}=\bar{\rho} \sum_{j=1}^{N}\left|h_{j, i}\right|^{2}=\bar{\rho}_{i}^{\prime} \sum_{j=1}^{N}\left|g_{j, i}\right|^{2}=\bar{\rho}_{i}^{\prime} \xi_{i}
$$

where $\bar{\rho}_{i}^{\prime}=\bar{\rho} L_{i}, \bar{\rho}=P_{t} / \sigma_{z}^{2}$ is the average SNR, and $\xi_{i}=\sum_{j=1}^{N}\left|g_{j, i}\right|^{2}$.

In practice, the CSI will be imperfect due to the channel estimation error. So in this paper, we will investigate the effect of imperfect CSI on the system performance with estimation errors modeled as complex Gaussian random variables [27]. We assume that the channel is perfectly known at the receiver, but there is error in the channel state information feedback from the receiver to the transmitter. The large-scale fading may be perfectly estimated due to very slow change. According to [27][28], the channel estimate of small-scale fading coefficients $\hat{\mathbf{g}}_{i}$ derived at the transmitter can be expressed as

$$
\hat{\mathbf{g}}_{i}=\mathbf{g}_{i}+\mathbf{e}_{i}
$$

where $\mathbf{g}_{i}, i=1, \ldots, M$, denotes the actual channel, $\mathbf{e}_{i}$ is channel error vector independent of $\mathbf{g}_{i}$. The elements of $\mathbf{e}_{i}$ are assumed to be independent complex Gaussian random variables with zero mean and variance $\sigma_{e}^{2}$, where $\sigma_{e}^{2}$ reflects the estimation quality. The larger the variance $\sigma_{e}^{2}$ is, the worse the estimation quality will be. When $\sigma_{e}^{2}=0$, the channel is perfectly known.

Based on the analysis above, the estimated SNR $\hat{\rho}_{i}$ between $R A_{i}$ and MT can be written as

$$
\hat{\rho}_{i}=\bar{\rho}_{i}^{\prime} \hat{\xi}_{i}=\bar{\rho}_{i}^{\prime} \sum_{j=1}^{N}\left|\hat{g}_{j, i}\right|^{2}
$$

where $\hat{\xi}_{i}=\sum_{j=1}^{N}\left|\hat{g}_{j, i}\right|^{2}, \hat{\boldsymbol{g}}_{j i}$ is the element of $\hat{\mathbf{g}}_{i}$. According to the Bayesian Linear Model and Theorem 10.3 [29], $\mathbf{g}_{i}$ conditioned on $\hat{\mathbf{g}}_{i}$ is a complex Gaussian vector, and the corresponding mean and covariance can be given by

$$
E\left\{\mathbf{g}_{i} \mid \hat{\mathbf{g}}_{i}\right\}=\left(1+\sigma_{e}^{2}\right)^{-1} \hat{\mathbf{g}}_{i}, \quad C\left\{\mathbf{g}_{i} \mid \hat{\mathbf{g}}_{i}\right\}=\sigma_{e}^{2}\left(1+\sigma_{e}^{2}\right)^{-1} \mathbf{I}_{N}
$$

where $C\{\cdot\}$ denotes the covariance. Utilizing Eq.(2.1-118) in [30], the PDF of $\xi_{i}$ given $\hat{\xi}_{i}$ is noncentral chi-square distributed, and can be expressed as

$$
f_{\xi_{i} \mid \hat{\xi}_{i}}(x \mid y)=\frac{\left(1+\sigma_{e}^{2}\right)^{N}}{\sigma_{e}^{2}}\left(\frac{x}{y}\right)^{(N-1) / 2} \exp \left(-\frac{y+\left(1+\sigma_{e}^{2}\right)^{2} x}{\sigma_{e}^{2}\left(1+\sigma_{e}^{2}\right)}\right) I_{N-1}\left(\frac{2 \sqrt{x y}}{\sigma_{e}^{2}}\right)
$$


where $I_{k}(\cdot)$ is the $k$ th-order modified Bessel function of the first kind [31]. With (6), by means of the transformation of variables, the conditional PDF of $\rho_{i}$ given $\hat{\boldsymbol{\rho}}_{\boldsymbol{i}}$ is obtained as

$$
f_{\rho_{i} \mid \hat{\rho}_{i}}(\rho \mid \hat{\rho})=\frac{\left(1+\sigma_{e}^{2}\right)^{N}}{\bar{\rho}_{i}^{\prime} \sigma_{e}^{2}}\left(\frac{\rho}{\hat{\rho}}\right)^{(N-1) / 2} \exp \left(-\frac{\hat{\rho}+\left(1+\sigma_{e}^{2}\right)^{2} \rho}{\bar{\rho}_{i}^{\prime} \sigma_{e}^{2}\left(1+\sigma_{e}^{2}\right)}\right) I_{N-1}\left(\frac{2 \sqrt{\rho \hat{\rho}}}{\bar{\rho}_{i}^{\prime} \sigma_{e}^{2}}\right)
$$

With (4), $\hat{\rho}_{\boldsymbol{i}}$ is central chi-square distributed with $2 N$, and thus its PDF and cumulative distribution function (CDF) can be, respectively, written as

$$
f_{\hat{\rho}_{i}}(\hat{\rho})=\frac{\hat{\rho}^{N-1}}{\left[\bar{\rho}_{i}^{\prime}\left(1+\sigma_{e}^{2}\right)\right]^{N} \Gamma(N)} \exp \left(-\hat{\rho} /\left(\bar{\rho}_{i}^{\prime}\left(1+\sigma_{e}^{2}\right)\right)\right)
$$

$$
\text { and } \quad F_{\hat{\rho}_{i}}(\hat{\rho})=1-\exp \left(-\hat{\rho} /\left(\bar{\rho}_{i}^{\prime}\left(1+\sigma_{e}^{2}\right)\right)\right) \sum_{n=0}^{N-1}\left(\hat{\rho} /\left(\bar{\rho}_{i}^{\prime}\left(1+\sigma_{e}^{2}\right)\right)\right)^{n} / n !
$$

where $\Gamma(\cdot)$ is Gamma function [31]. As analyzed in section 2, the selective diversity scheme is applied to the transmitter, i.e., based on the estimation information feedback at the transmitter, one 'best' RA is selected for transmission to maximize the estimated SNR. Thus, we have:

$$
\hat{\rho}=\max \left\{\hat{\rho}_{1}, \ldots, \hat{\rho}_{M}\right\}
$$

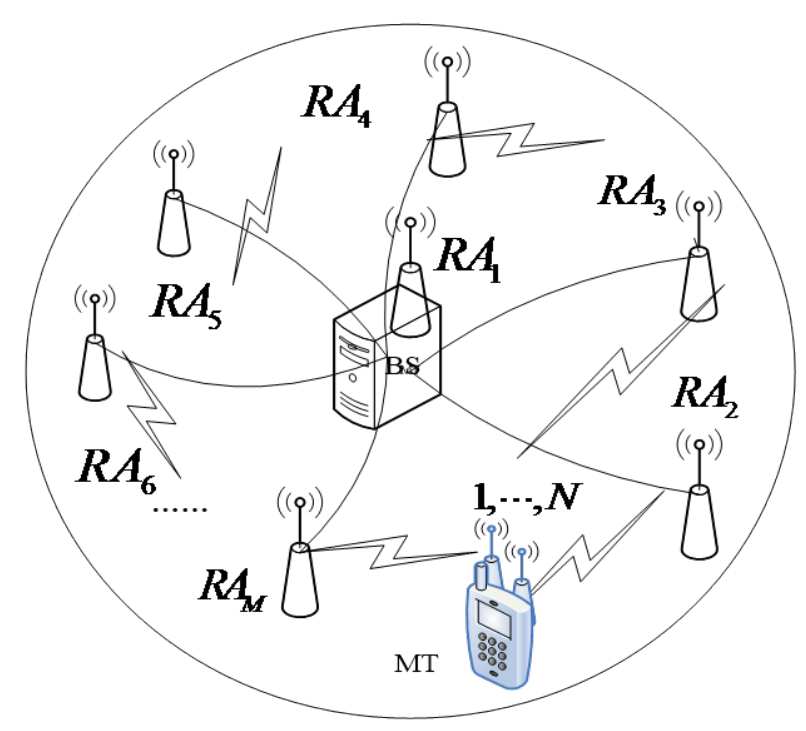

Fig. 1. Diagram of DAS structure.

It is reasonable to assume that $\left\{\hat{\rho}_{i}\right\}$ are independent of each other due to the large space among remote antennas. Hence, using (9) and [32, (47)], the CDF of $\hat{\rho}, F(\hat{\rho})$, can be obtained as

$$
F_{\hat{\rho}}(\hat{\rho})=\prod_{i=1}^{M} F_{\hat{\rho}_{i}}(\hat{\rho})=\prod_{i=1}^{M}\left[1-\exp \left(-\frac{\hat{\rho}}{\bar{\rho}_{i}^{\prime}\left(1+\sigma_{e}^{2}\right)}\right) \sum_{n=0}^{N-1} \frac{1}{n !}\left(\frac{\hat{\rho}}{\bar{\rho}_{i}^{\prime}\left(1+\sigma_{e}^{2}\right)}\right)^{n}\right]
$$

With (11), the PDF of $\hat{\rho}$ can be given by 


$$
f_{\hat{\rho}}(\hat{\rho})=\sum_{i=1}^{M} f_{\hat{\rho}_{i}}(\hat{\rho}) \prod_{m=1, \neq i}^{M} F_{\hat{\rho}_{m}}(\hat{\rho})=\sum_{i=1}^{M} \frac{\beta_{i}^{N}}{\Gamma(N)} \sum_{p=0}^{M-1}(-1)^{p} \sum_{\tilde{\tau}(p, M-1)} \exp \left(-\hat{\rho}\left(\beta_{i}+\sum_{v=1, \neq i}^{M} l_{v} \beta_{v}\right)\right) \sum_{u=0}^{\tilde{U}} \tilde{A}_{u, M-1} \hat{\rho}^{u+N-1}
$$

where $\beta_{i}=\left[\bar{\rho}_{i}^{\prime}\left(1+\sigma_{e}^{2}\right)\right]^{-1}, \tilde{\tau}(p, M-1)$ is the set of $(M-1)$-tuples such that $\tilde{\tau}(p, M-1)=$

$$
\begin{aligned}
& \left\{\left(l_{1}, \ldots, l_{m-1}, l_{m+1}, \ldots l_{M}\right): l_{q} \in\{0,1\}, \sum_{q=1, \neq m}^{M} l_{q}=p\right\}, \tilde{U}=\sum_{v=1, \neq m}^{M} K_{v}, K_{v}=l_{v}(N-1), \\
& \tilde{\omega}(u, M-1)=\left\{\left(k_{1}, \ldots k_{m-1}, k_{m+1}, \ldots k_{M}\right): k_{v} \in\left\{0,1, \ldots, K_{v}\right\}, \sum_{v=1, \neq m}^{M} k_{v}=u\right\}, \tilde{A}_{u, M-1}=\sum_{\tilde{\omega}(u, M-1)} \prod_{v=1, \neq m}^{M} \frac{\left(\beta_{v}\right)^{k_{v}}}{k_{v} !} .
\end{aligned}
$$

\section{Ergodic Capacity of DAS}

In this section, we will give the PDF derivation of effective SNR and ergodic capacity analysis of DAS in the presence of imperfect CSI. Firstly, the PDF of effective output SNR of the system is derived. Let $\rho$ denotes the effective output SNR and $a=\underset{i \in M}{\arg \max }\left\{\rho_{i}\right\}$, then with (7), (8) and (9), the PDF of $\rho$ can be expressed as

$$
\begin{aligned}
f_{\rho}(\rho) & =\sum_{i=1}^{M} \int_{0}^{\infty} f_{\rho_{i} \mid \hat{\rho}_{i}}\left(\rho \mid \hat{\rho}_{i}=\hat{\rho}, a=i\right) P_{r o b}\left(a=i \mid \hat{\rho}_{i}=\hat{\rho}\right) f_{\hat{\rho}_{i}}\left(\hat{\rho}_{i}=\hat{\rho}\right) d \hat{\rho} \\
& =\sum_{i=1}^{M} \int_{0}^{\infty} f_{\rho_{i} \mid \hat{\rho}_{i}}(\rho \mid \hat{\rho}) f_{\hat{\rho}_{i}}(\hat{\rho}) \prod_{m=1, \neq i}^{M} F_{\hat{\rho}_{m}}(\hat{\rho}) d \hat{\rho} d \rho
\end{aligned}
$$

where $\quad P_{\text {rob }}\left(a=i \mid \hat{\rho}_{i}=\hat{\rho}\right) f_{\hat{\rho}_{i}}\left(\hat{\rho}_{i}=\hat{\rho}\right)=f_{\hat{\rho}_{i}}(\hat{\rho}) \prod_{m=1, \neq i}^{M} F_{\hat{\rho}_{m}}(\hat{\rho}) \quad[33] \quad$ and $\quad$ the conditional PDF $f_{\rho_{i} \mid \hat{\hat{p}}_{i}}\left(\rho \mid \hat{\rho}_{i}=\hat{\rho}, a=i\right)=f_{\rho_{i} \mid \hat{\hat{\rho}_{i}}}(\rho \mid \hat{\rho})$ is defined as (7).

Substituting (7)-(9) into (13), and using (12), (13) can written as

$$
\begin{aligned}
& f_{\rho}(\rho)=\sum_{i=1}^{M} \int_{0}^{\infty} \frac{\left(1+\sigma_{e}^{2}\right)^{N}}{\bar{\rho}_{i}^{\prime} \sigma_{e}^{2}}\left(\frac{\rho}{\hat{\rho}}\right)^{(N-1) / 2} \exp \left(-\frac{\hat{\rho}+\left(1+\sigma_{e}^{2}\right)^{2} \rho}{\bar{\rho}_{i}^{\prime} \sigma_{e}^{2}\left(1+\sigma_{e}^{2}\right)}\right) I_{N-1}\left(\frac{2 \sqrt{\rho \hat{\rho}}}{\bar{\rho}_{i}^{\prime} \sigma_{e}^{2}}\right) \\
& \times \frac{\beta_{i}^{N}}{\Gamma(N)} \sum_{p=0}^{M-1}(-1)^{p} \sum_{\tilde{\tau}(p, M-1)} \exp \left(-\hat{\rho}\left(\beta_{i}+\sum_{v=1, \neq i}^{M} l_{v} \beta_{v}\right)\right) \sum_{u=0}^{\tilde{U}} \tilde{A}_{u, M-1} \hat{\rho}^{u+N-1} d \hat{\rho} \\
&=\sum_{i=1}^{M} \frac{\left(1+\sigma_{e}^{2}\right)^{N} \rho^{(N-1) / 2}}{\bar{\rho}_{i}^{\prime} \sigma_{e}^{2}} \exp \left(-\frac{\left(1+\sigma_{e}^{2}\right) \rho}{\bar{\rho}_{i}^{\prime} \sigma_{e}^{2}}\right) \sum_{p=0}^{M-1}(-1)^{p} \sum_{\tilde{\tau}(p, M-1)} \frac{\beta_{i}^{N}}{\Gamma(N)} \sum_{u=0}^{\tilde{U}} \tilde{A}_{u, M-1} \\
& \quad \times \int_{0}^{\infty} \hat{\rho}^{u+(N-1) / 2} \exp \left(-\hat{\rho}\left(\beta_{i}+\sum_{v=1, \neq i}^{M} l_{v} \beta_{v}+\beta_{i} \sigma_{e}^{-2}\right)\right) I_{N-1}\left(2 \sqrt{\rho \hat{\rho}} /\left(\bar{\rho}_{i}^{\prime} \sigma_{e}^{2}\right)\right) d \hat{\rho}
\end{aligned}
$$

Utilizing Eqs.(6.643.4) and (8.406.3) and Eq.(8.970.1) in [31], (14) can be simplified as 


$$
\begin{aligned}
f_{\rho}(\rho)= & \sum_{i=1}^{M} \frac{\rho^{(N-1) / 2}}{\bar{\rho}_{i}^{\prime} \sigma_{e}^{2}} \exp \left(-\frac{\left(1+\sigma_{e}^{2}\right) \rho}{\bar{\rho}_{i}^{\prime} \sigma_{e}^{2}}\right) \sum_{p=0}^{M-1}(-1)^{p} \sum_{u=0}^{\tilde{U}} \tilde{A}_{u, M-1} \sum_{\tilde{\tau}(p, M-1)} \frac{1}{\Gamma(N) \bar{\rho}_{i}^{\prime N}} \\
& \times \sum_{t=0}^{u} \frac{u !}{t !}\left(\begin{array}{c}
u+N-1 \\
u-t
\end{array}\right) \frac{\rho^{t+(N-1) / 2}\left(\bar{\rho}_{i}^{\prime} \sigma_{e}^{2}\right)^{u-t+1}}{\left[\left(\bar{\rho}_{i}^{\prime} \sigma_{e}^{2}\right) \sum_{v=1, \neq i}^{M} l_{v} \beta_{v}+1\right]^{u+N+t}} \exp \left(\left(\bar{\rho}_{i}^{\prime} \sigma_{e}^{2}\right)^{2} \sum_{v=1, \neq i}^{M} l_{v} \beta_{v}+\bar{\rho}_{i}^{\prime} \sigma_{e}^{2}\right)
\end{aligned}
$$

By further simplification, Eq.(15) can be expressed as follows:

$$
f_{\rho}(\rho)=\sum_{i=1}^{M} \frac{\bar{\rho}_{i}^{\prime-N}}{\Gamma(N)} \sum_{p=0}^{M-1} \sum_{\tilde{\tau}(p, M-1)} \sum_{u=0}^{\tilde{U}} \sum_{t=0}^{u} \frac{u !}{t !}\left(\begin{array}{c}
u+N-1 \\
u-t
\end{array}\right) \frac{(-1)^{p} \tilde{A}_{u, M-1} \rho^{t+N-1}\left(\bar{\rho}_{i}^{\prime} \sigma_{e}^{2}\right)^{u-t}}{\left[\left(\bar{\rho}_{i}^{\prime} \sigma_{e}^{2}\right) \sum_{v=1, \neq i}^{M} l_{v} \beta_{v}+1\right]^{u+N+t}} \exp \left(-\rho \lambda_{i}\right)
$$

where $\lambda_{i}=\left(1 / \bar{\rho}_{i}^{\prime}\right)+\sum_{v=1, \neq i}^{M} l_{v} \beta_{v} /\left(1+\bar{\rho}_{i}^{\prime} \sigma_{e}^{2} \sum_{v=1, \neq i}^{M} l_{v} \beta_{v}\right)$. This is PDF of the effective output SNR of the system. When $\sigma_{e}{ }^{2}=0$ (corresponding to perfect CSI), (16) is reduced to

$$
f_{\rho}(\rho)=\sum_{i=1}^{M} \frac{\bar{\rho}_{i}^{\prime-N}}{\Gamma(N)} \sum_{p=0}^{M-1} \sum_{\tilde{\tau}(p, M-1)} \sum_{u=0}^{\tilde{U}}(-1)^{p} \tilde{A}_{u, M-1} \rho^{u+N-1} \exp \left(-\rho\left(\frac{1}{\bar{\rho}_{i}^{\prime}}+\sum_{v=1, \neq i}^{M} \frac{l_{v}}{\bar{\rho}_{v}^{\prime}}\right)\right)
$$

Equation (17) is the PDF of the effective SNR of the system under perfect CSI, which is the same as (12) for $\sigma_{e}{ }^{2}=0$. Namely, under perfect CSI, their PDFs are identical as expected.

In what follows, we will give the derivation of ergodic capacity. Ergodic capacity is an appropriate capacity metric for channel that varies quickly, or where the channel is ergodic over the time period of interest [34], it is equal to Shannon capacity in an Additive White Gaussian Noise (AWGN) channel with $\log _{2}(1+\rho)$, averaged over the distribution of $\rho$, here we consider capacity per unit bandwidth (in bits/s/Hz). Thus, the corresponding ergodic capacity is expressed as

$$
C_{a}=E\{C\}=E\left\{\log _{2}(1+\rho)\right\}=(1 / \ln 2) \int_{0}^{\infty} f(\rho) \ln (1+\rho) d \rho
$$

Substituting (16) into (18) yields

$$
\begin{aligned}
C_{a}= & \sum_{i=1}^{M} \frac{\bar{\rho}_{i}^{\prime-N}}{\Gamma(N) \ln 2} \sum_{p=0}^{M-1} \sum_{\tilde{\tau}(p, M-1)} \sum_{u=0}^{\tilde{U}} \sum_{t=0}^{u} \frac{u !}{t !}\left(\begin{array}{c}
u+N-1 \\
u-t
\end{array}\right) \\
& \times \int_{0}^{\infty} \frac{(-1)^{p} \tilde{A}_{u, M-1}\left(\bar{\rho}_{i}^{\prime} \sigma_{e}^{2}\right)^{u-t}}{\left[\left(\bar{\rho}_{i}^{\prime} \sigma_{e}^{2}\right) \sum_{v=1, \neq i}^{M} l_{v} \beta_{v}+1\right]^{u+N+t}} \rho^{t+N-1} \ln (1+\rho) e^{-\rho \lambda_{i}} d \rho
\end{aligned}
$$

According to the results in Appendix, we have:

$$
J=\int_{0}^{\infty} \rho^{t+N-1} \ln (1+\rho) e^{-\rho \lambda_{i}} d \rho=\frac{\Gamma(t+N)}{\lambda_{i}^{t+N}}\left\{\mathcal{P}_{t+N}\left(-\lambda_{i}\right) E_{1}\left(\lambda_{i}\right)+\sum_{k=1}^{t+N-1} \frac{\mathcal{P}_{t+N-k}\left(-\lambda_{i}\right) \mathcal{P}_{k}\left(\lambda_{i}\right)}{k}\right\}
$$

where $E_{1}(\cdot)$ denotes the first order exponential integral function, $\mathcal{P}_{n}(x)=\sum_{l=0}^{n-1} x^{l} e^{-x} / l$ ! is the Poisson distribution [31].

Substituting (20) into (19) gives 


$$
\begin{aligned}
C_{a}= & \sum_{i=1}^{M} \frac{\bar{\rho}_{i}^{\prime-N}}{\Gamma(N) \ln 2} \sum_{p=0}^{M-1} \sum_{\tilde{\tau}(p, M-1)} \sum_{u=0}^{\tilde{U}} \sum_{t=0}^{u} \frac{u !}{t !}\left(\begin{array}{c}
u+N-1 \\
u-t
\end{array}\right) \frac{(-1)^{p} \tilde{A}_{u, M-1}\left(\bar{\rho}_{i}^{\prime} \sigma_{e}^{2}\right)^{u-t}}{\left[\left(\bar{\rho}_{i}^{\prime} \sigma_{e}^{2}\right) \sum_{v=1, \neq i}^{M} l_{v} \beta_{v}+1\right]^{u+N+t}} \\
& \times \frac{\Gamma(t+N)}{\lambda_{i}^{t+N}}\left\{\mathcal{P}_{t+N}\left(-\lambda_{i}\right) E_{1}\left(\lambda_{i}\right)+\sum_{k=1}^{t+N-1} \frac{\mathcal{P}_{t+N-k}\left(-\lambda_{i}\right) \mathcal{P}_{k}\left(\lambda_{i}\right)}{k}\right\}
\end{aligned}
$$

Eq.(21) is an accurate closed-form expression of ergodic capacity of DAS under imperfect CSI, and can agree the simulation very well. When the CSI is perfectly known with $\sigma_{e}^{2}=0,(21)$ is reduced to

$$
\begin{aligned}
C_{a} & =\sum_{i=1}^{M} \sum_{p=0}^{M-1} \sum_{\tilde{\tau}(p, M-1)} \sum_{u=0}^{\tilde{U}} \frac{(-1)^{p} \tilde{A}_{u, M-1} \Gamma(u+N)}{\Gamma(N) \ln 2 \lambda_{i}^{u+N} \bar{\rho}_{i}^{\prime N}} \\
& \times\left\{\mathcal{P}_{u+N}\left(-\lambda_{i}\right) E_{1}\left(\lambda_{i}\right)+\sum_{k=1}^{u+N-1} \mathcal{P}_{u+N-k}\left(-\lambda_{i}\right) \mathcal{P}_{k}\left(\lambda_{i}\right) / k\right\}
\end{aligned}
$$

where $\lambda_{i}=\left(1 / \bar{\rho}_{i}^{\prime}\right)+\sum_{v=1, \neq i}^{M}\left(l_{v} / \bar{\rho}_{v}^{\prime}\right)$, equation (22) is an accurate closed-form expression of ergodic capacity of DAS under perfect CSI.

Besides, for single receive antenna, $N=1$, the corresponding $K_{v}=l_{v}(N-1)=0, \tilde{U}=\sum_{v=1, \neq m}^{M} K_{v}=0$, and resulting $u=0$ in (21). Thus, (21) is reduced to

$$
\begin{aligned}
C_{a} & =\sum_{i=1}^{M} \frac{\bar{\rho}_{i}^{\prime-1}}{\ln 2} \sum_{p=0}^{M-1} \sum_{\tilde{\tau}(p, M-1)} \frac{(-1)^{p}}{\left(\bar{\rho}_{i}^{\prime} \sigma_{e}^{2}\right) \sum_{v=1, \neq i}^{M} l_{v} \beta_{v}+1} \frac{1}{\lambda_{i}} e^{\lambda_{i}} E_{1}\left(\lambda_{i}\right) \\
& =\frac{1}{\ln 2} \sum_{i=1}^{M} \sum_{p=0}^{M-1} \sum_{\tilde{\tau}(p, M-1)} \frac{(-1)^{p}}{1+\left(1+\sigma_{e}^{2}\right) \bar{\rho}_{i}^{\prime} \sum_{v=1, \neq i}^{M} l_{v} \beta_{v}} e^{\lambda_{i}} E_{1}\left(\lambda_{i}\right)
\end{aligned}
$$

Based on the analysis above, the derived (21) includes the ones under perfect CSI and single receive antenna as special cases.

\section{Outage capacity of DAS}

The outage capacity and probability are important performance measure of communication systems in fading channels [21][22][23][24]. However, the related research is relatively less, especially the analysis under imperfect CSI is much less. For this reason, we will give the outage performance analysis of the DAS in the presence of imperfect CSI, and derive the closed-form outage capacity and probability. Since the channel capacity is a random variable, it is meaningful to consider its statistical distribution. A useful measure of statistical characteristic is the outage capacity [35]. For the given outage capacity $C_{o}, P\left(C_{o}\right)$ is defined as the outage probability that the channel capacity $C=\log _{2}(1+\rho)$ falls below $C_{0}$. Thus, the outage probability can be expressed as

$$
P\left(C_{o}\right)=\operatorname{Pr}\left(C \leq C_{o}\right)=\operatorname{Pr}\left(\rho \leq 2^{C_{o}}-1\right)=F_{\rho}\left(\rho_{t h}\right)
$$

where $\rho_{\text {th }}=2^{C_{o}}-1$ is the outage threshold, $F_{\rho}(\rho)$ is the CDF of $\rho$ and can be obtained from (16) as follows: 


$$
F_{\rho}(\rho)=\sum_{i=1}^{M} \frac{\bar{\rho}_{i}^{\prime-N}}{\Gamma(N)} \sum_{p=0}^{M-1} \sum_{\tilde{\tau}(p, M-1)} \sum_{u=0}^{\tilde{U}} \sum_{t=0}^{u} \frac{u !}{t !}\left(\begin{array}{c}
u+N-1 \\
u-t
\end{array}\right) \frac{(-1)^{p} \tilde{A}_{u, M-1}\left(\bar{\rho}_{i}^{\prime} \sigma_{e}^{2}\right)^{u-t} \gamma\left(t+N, \rho \lambda_{i}\right)}{\left[\left(\bar{\rho}_{i}^{\prime} \sigma_{e}^{2}\right) \sum_{v=1, \neq i}^{M} l_{v} \beta_{v}+1\right]^{u+N+t} \lambda_{i}^{t+N}}
$$

where $\gamma(a, v) \triangleq \int_{0}^{v} t^{a-1} e^{-t} d t$ is the lower incomplete Gamma function [31]. Substituting (25) into (24) yields

$$
P\left(C_{o}\right)=\sum_{i=1}^{M} \frac{\bar{\rho}_{i}^{\prime-N}}{\Gamma(N)} \sum_{p=0}^{M-1} \sum_{\tilde{\tau}(p, M-1)} \sum_{u=0}^{\tilde{U}} \sum_{t=0}^{u} \frac{u !}{t !}\left(\begin{array}{c}
u+N-1 \\
u-t
\end{array}\right) \frac{(-1)^{p} \tilde{A}_{u, M-1}\left(\bar{\rho}_{i}^{\prime} \sigma_{e}^{2}\right)^{u-t} \gamma\left(t+N,\left(2^{C_{o}}-1\right) \lambda_{i}\right)}{\left[\left(\bar{\rho}_{i}^{\prime} \sigma_{e}^{2}\right) \sum_{v=1, \neq i}^{M} l_{v} \beta_{v}+1\right]^{u+N+t} \lambda_{i}^{t+N}}
$$

Equation (26) is an accurate closed-form expression of outage probability of DAS with imperfect CSI for the given outage capacity $C_{o}$, which is shown to accord with the simulation. When $\sigma_{e}{ }^{2}=0$, (26) is reduced to

$$
P\left(C_{o}\right)=\sum_{i=1}^{M} \frac{\bar{\rho}_{i}^{\prime-N}}{\Gamma(N)} \sum_{p=0}^{M-1} \sum_{\tilde{\tau}(p, M-1)} \sum_{u=0}^{\tilde{U}}(-1)^{p} \tilde{A}_{u, M-1} \gamma\left(N,\left(2^{C_{0}}-1\right) \lambda_{i}\right) / \lambda_{i}^{N}
$$

Eq.(27) is an accurate closed-form expression of outage probability of DAS with perfect CSI. Besides, for single receive antenna, $N=1$, (26) is reduced to the following (28).

$$
P\left(C_{o}\right)=\sum_{i=1}^{M} \frac{1}{\bar{\rho}^{\prime}} \sum_{p=0}^{M-1} \sum_{\tilde{\tau}(p, M-1)} \frac{(-1)^{p}\left[1-\exp \left(-\left(2^{C_{0}}-1\right) \lambda_{i}\right)\right]}{\left[\left(\bar{\rho}_{i}^{\prime} \sigma_{e}^{2}\right) \sum_{v=1, \neq i}^{M} l_{v} \beta_{v}+1\right] \lambda_{i}}
$$

This is an accurate closed-form expression of outage probability of DAS with single receive antenna under imperfect CSI.

For a given outage probability $\varepsilon=P\left(C_{o}\right)$, using (26) or (27) to calculate the outage capacity $C_{o}$ directly will be difficult. For this reason, we propse a practical iterative algorithm based on Newton's method to obtain $C_{o}$. With (26), we express solving the outage capacity as finding the root of $\phi\left(C_{0}\right)=0$ with

$$
\begin{aligned}
& \phi\left(C_{o}\right)=F_{\rho}\left(\rho_{t h}\right)-\varepsilon=F_{\rho}\left(2^{C_{o}}-1\right)-\varepsilon \\
& =\sum_{i=1}^{M} \frac{\bar{\rho}_{i}^{\prime-N}}{\Gamma(N)} \sum_{p=0}^{M-1} \sum_{\tilde{\tau}(p, M-1)} \sum_{u=0}^{\tilde{U}} \sum_{t=0}^{u} \frac{u !}{t !}\left(\begin{array}{c}
u+N-1 \\
u-t
\end{array}\right) \frac{(-1)^{p} \tilde{A}_{u, M-1}\left(\bar{\rho}_{i}^{\prime} \sigma_{e}^{2}\right)^{u-t} \gamma\left(t+N,\left(2^{C_{o}}-1\right) \lambda_{i}\right)}{\left[\left(\bar{\rho}_{i}^{\prime} \sigma_{e}^{2}\right) \sum_{v=1, \neq i}^{M} l_{v} \beta_{v}+1\right]^{u+N+t} \lambda_{i}^{t+N}}-\varepsilon
\end{aligned}
$$

Differentiating $\phi\left(C_{0}\right)$ with respect to $C_{o}$ gives

$$
\phi^{\prime}\left(C_{o}\right)=\partial \phi\left(C_{o}\right) / \partial C_{o}=f_{\rho}\left(2^{C_{o}}-1\right) 2^{C_{o}} \ln 2>0
$$

This is because $\ln 2,2^{C_{o}}$ and the PDF $f_{\rho}\left(2^{C_{o}}-1\right)$ are all positive. So the derivative $\phi^{\prime}\left(C_{o}\right)$ is positive, and accordingly, $\phi\left(C_{0}\right)$ is a strictly monotonically increasing function of $C_{o}$. With (29), and considering the outage probability $\varepsilon \in(0,1)$, we have:

$$
\phi(0)=0-\varepsilon=-\varepsilon<0 \text {, and } \phi(\infty)=\lim _{C_{o} \rightarrow \infty} \phi\left(C_{o}\right)=1-\varepsilon>0
$$

According to the analysis above, the equation $\phi\left(C_{0}\right)=0$ is shown to have a unique solution for $C_{o}>0$. Substituting (16) into (30) yields 


$$
\begin{aligned}
\phi^{\prime}\left(C_{o}\right)=\sum_{i=1}^{M} \frac{\bar{\rho}_{i}^{\prime-N}}{\Gamma(N)} \sum_{p=0}^{M-1} \sum_{\tilde{\tau}(p, M-1)} \sum_{u=0}^{\tilde{U}} \sum_{t=0}^{u} \frac{u !}{t !}\left(\begin{array}{c}
u+N-1 \\
u-t
\end{array}\right) \frac{(-1)^{p} \tilde{A}_{u, M-1}\left(\bar{\rho}_{i}^{\prime} \sigma_{e}^{2}\right)^{u-t}}{\left[\left(\bar{\rho}_{i}^{\prime} \sigma_{e}^{2}\right) \sum_{v=1, \neq i}^{M} l_{v} \beta_{v}+1\right]^{u+N+t}} \\
\times\left(2^{C_{o}}-1\right)^{t+N-1} \exp \left(-\left(2^{C_{o}}-1\right) \lambda_{i}\right) 2^{C_{o}} \ln 2
\end{aligned}
$$

There are many methods such as bisection for finding the root of a strictly monotonic function. We propose to use Newton's method to find the root iteratively because it has the quadratic convergence rate. Newton's method is described as follows.

$$
C_{o, k+1}=C_{o, k}-\phi\left(C_{o, k}\right) / \phi^{\prime}\left(C_{o, k}\right)
$$

where $C_{o, k}$ is the $k$-th iterative value of outage capacity. $\phi\left(C_{o, k}\right)$ and $\phi^{\prime}\left(C_{o, k}\right)$ are calculated by (29) and (32), respectively. With (33), the accurate outage capacity can be obtained, and may agree the simulation well.

To avoid the above iterative calculation, we also provide a closed-form expression for computing $C_{0}$. With (16), using the upper incomplete Gamma function $\Gamma(\cdot, \cdot)$, and let $x=1+\rho$, then the second moment of the channel capacity $C=\log _{2}(1+\rho)$ can be given by

$$
\begin{aligned}
E\left(C^{2}\right)= & \int_{0}^{+\infty}\left(\log _{2}(1+\rho)\right)^{2} f(\rho) d \rho=\int_{1}^{+\infty}\left(\log _{2} x\right)^{2} f_{\rho}(x-1) d x \\
= & \sum_{i=1}^{M} \frac{\bar{\rho}_{i}^{\prime-N}}{\Gamma(N)} \sum_{p=0}^{M-1} \sum_{\tilde{\tau}(p, M-1)} \sum_{u=0}^{\tilde{U}} \sum_{t=0}^{u} \frac{u !}{t !}\left(\begin{array}{c}
u+N-1 \\
u-t
\end{array}\right) \frac{(-1)^{p} \tilde{A}_{u, M-1}\left(\bar{\rho}_{i}^{\prime} \sigma_{e}^{2}\right)^{u-t}}{\left[\left(\bar{\rho}_{i}^{\prime} \sigma_{e}^{2}\right) \sum_{v=1, \neq i}^{M} l_{v} \beta_{v}+1\right]^{u+N+t}} \\
& \times \frac{2 e^{\lambda_{i}}}{(\ln 2)^{2}} \sum_{q=0}^{N+t-1}(-1)^{q}\left(\begin{array}{c}
N+t-1 \\
q
\end{array}\right) \lambda_{i}^{q-t-N} \int_{1}^{\infty} x^{-1}(\ln x) \Gamma\left(t+N-q, \lambda_{i} x\right) d x
\end{aligned}
$$

Using Meijer's $G$-function and Eq.(7.831) in [31], (34) can be simplified as

$$
\begin{aligned}
E\left(C^{2}\right)= & \sum_{i=1}^{M} \frac{\bar{\rho}_{i}^{\prime-N}}{\Gamma(N)} \sum_{p=0}^{M-1} \sum_{\tilde{\tau}(p, M-1)} \sum_{u=0}^{\tilde{U}} \sum_{t=0}^{u} \frac{u !}{t !}\left(\begin{array}{c}
u+N-1 \\
u-t
\end{array}\right) \frac{(-1)^{p} \tilde{A}_{u, M-1}\left(\bar{\rho}_{i}^{\prime} \sigma_{e}^{2}\right)^{u-t}}{\left[\left(\bar{\rho}_{i}^{\prime} \sigma_{e}^{2}\right) \sum_{v=1, \neq i}^{M} l_{v} \beta_{v}+1\right]^{u+N+t}} \\
& \times \frac{2 e^{\lambda_{i}}}{(\ln 2)^{2}} \sum_{q=0}^{N+t-1}(-1)^{q}\left(\begin{array}{c}
N+t-1 \\
q
\end{array}\right) \lambda_{i}^{q-t-N} G_{3,4}^{4,0}\left(\lambda_{i} \mid \begin{array}{cc}
1, & 1, \quad 1 \\
0,0, N+t-q, 0
\end{array}\right)
\end{aligned}
$$

From (35) and (21), the variance of the channel capacity $C$ can be written as

$$
V_{c}=E\left(C^{2}\right)-[E(C)]^{2}=E\left(C^{2}\right)-C_{a}^{2}
$$

With the mean value (21) and the variance (36), using a Gaussian distribution approximation, the CDF of $C$ can be approximated as

$$
F_{C}(c) \approx \int_{-\infty}^{c} \frac{1}{\sqrt{2 \pi V_{c}}} \exp \left(-\frac{\left(x-C_{a}\right)^{2}}{2 V_{c}}\right) d x=1-0.5 \operatorname{erf}\left\{\frac{c-C_{a}}{\sqrt{2 V_{c}}}\right\}
$$

where $\operatorname{erf} c\{\cdot\}$ denotes the complementary error function [31]. By setting the above $F_{C}(c)$ equal to the given outage probability $\varepsilon\left(\right.$ i.e., $F_{C}(c)=\varepsilon$ ), the outage capacity $C_{o}$ can be calculated from (37) as

$$
C_{o} \approx \sqrt{2 V_{c}} \operatorname{erfc}^{-1}\{2(1-\varepsilon)\}+C_{a}
$$


where $\operatorname{erfc}^{-1}\{$. $\}$ denotes the inverse complementary error function. Eq. (38) is a closed-form expression of outage capacity, and may obtain the value close to the actual simulation. Moreover, from (38) and (26), it is found that $C_{o}$ will increase as $\varepsilon$ increases, and decrease as $\varepsilon$ decreases, as expected. Hence, the derived expressions may provide a good method to assess the outage performance of DAS under imperfect CSI. Besides, (38) is relatively simpler because it has closed-form expression and does not need any iterative calculation.

\section{Simulation results}

In this section, we use the derived theoretical performance formulae and computer simulation to evaluate the performance of the DAS over composite Rayleigh channels including path loss. In simulation, the cell shape is assumed to be circle with radius $R$ for analysis convenience. The BS $\left(R A_{1}\right)$ is in the center of the cell, and the other $M-1$ RAs are uniformly distributed over a circle with radius $r$. Correspondingly, the polar coordinate of the $i$-th RA is $(r, 2 \pi(i-1) /(M-1))$, $i=2,3, \ldots, M$. Unless otherwise specified, the main parameters are listed as: $M=7 ; r=2 R / 3$; the reference distance $d_{r}=80 \mathrm{~m}$; radius of the cell $R=1000 \mathrm{~m}$. The simulation results are illustrated in Figs. 2-7.

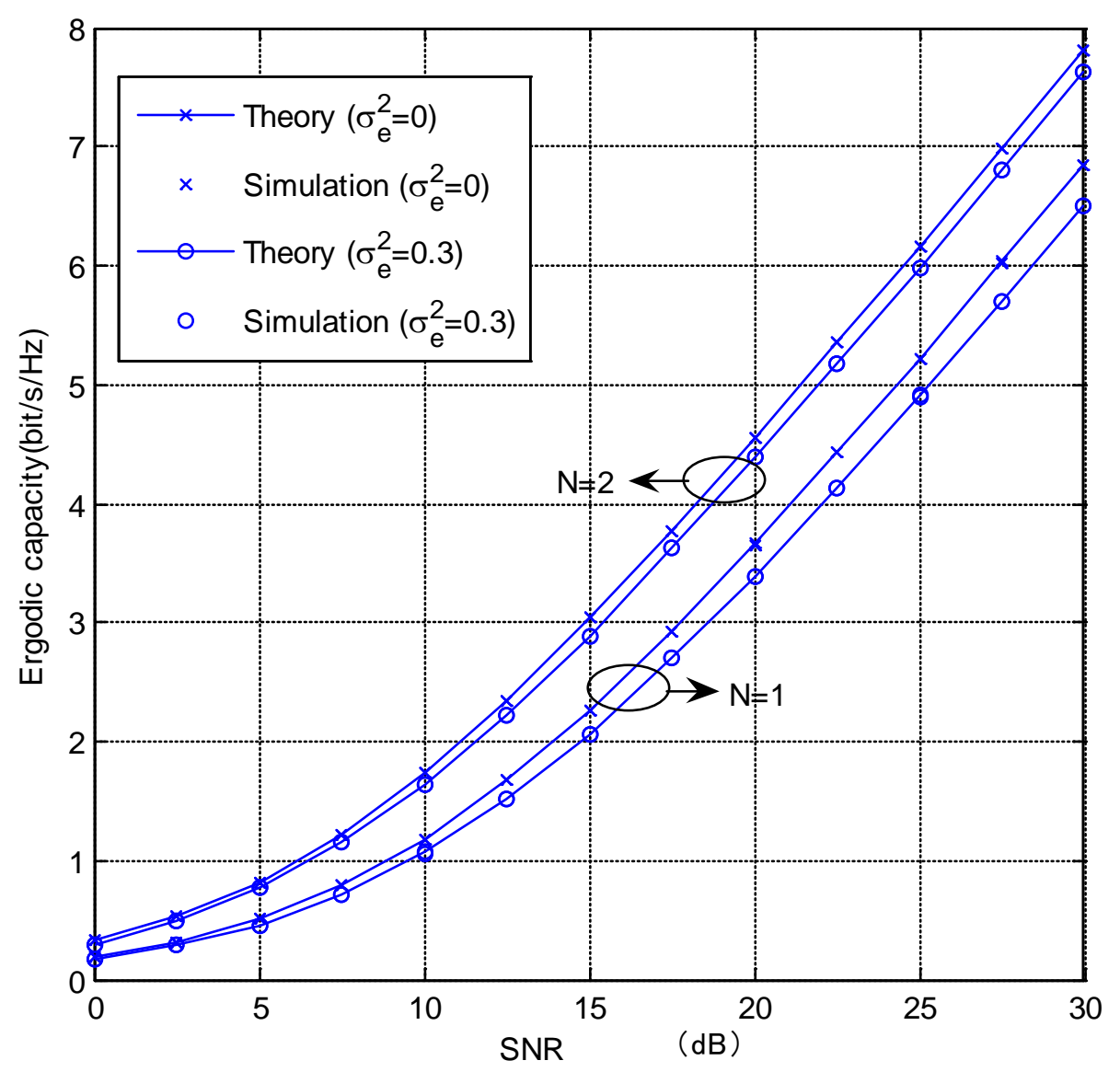

Fig. 2. Ergodic capacity of DAS with different receive antennas and estimation errors 
In Fig. 2, we plot the theoretical ergodic capacity and corresponding simulation of the DAS with different receive antennas and estimation errors, where path loss exponent $\alpha=2, N=1,2$, perfect CSI $\left(\sigma_{e}{ }^{2}=0\right)$ and imperfect CSI $\left(\sigma_{e}{ }^{2}=0.3\right)$ are compared. The theoretical ergodic capacity is calculated by (21). As shown in Fig. 2, the theoretical capacities are in good agreement with the simulated ones for different estimation errors and receive antennas. Moreover, due to the estimation error, the capacity under imperfect CSI $\left(\sigma_{e}{ }^{2}=0.3\right)$ is lower than that under perfect CSI $\left(\sigma_{e}{ }^{2}=0\right)$ as expected. Besides, it is observed that the DAS with two receive antennas outperforms that with single receive antenna because of greater diversity, which accords with the existing knowledge. The above results show that the derived ergodic capacity is valid.

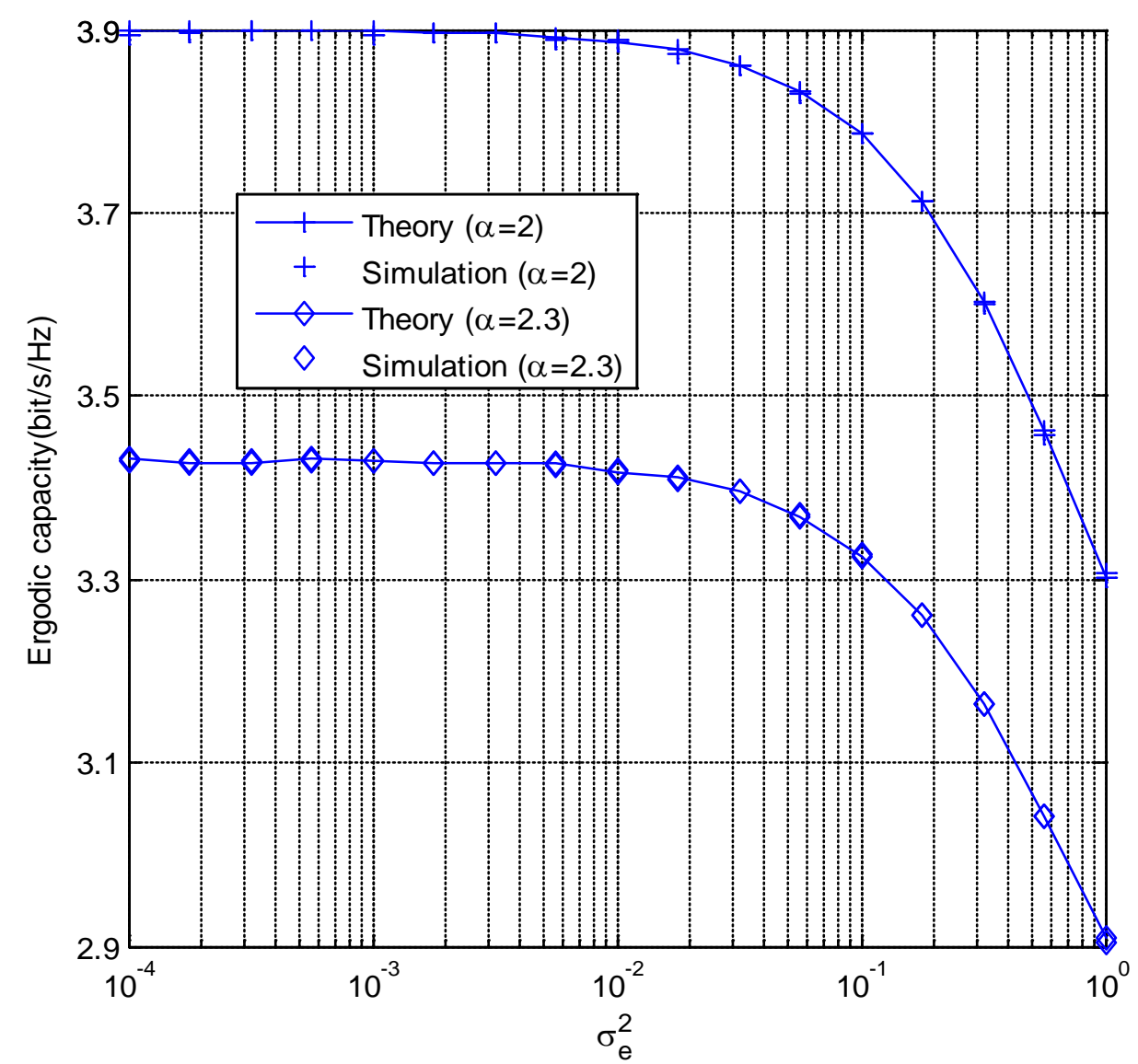

Fig. 3. Ergodic capacity versus estimation error variance for DAS with different path loss exponents

In Fig. 3, the effect of the estimation error on the capacity is evaluated, and the ergodic capacity versus channel estimation error variance $\left(\sigma_{e}{ }^{2}\right)$ for DAS with imperfect CSI is plotted, where nine RAs and single receive antenna are considered. The average SNR is set equal to $20 \mathrm{~dB}$, path loss exponent $\alpha=2$ and 2.3. From Fig. 3, it is found that the theoretical capacity can agree the corresponding simulation very well due to its accuracy. The ergodic capacity of DAS 
with $\alpha=2.3$ is lower than that with $\alpha=2$ because of large path loss as expected. Besides, as the estimation error increases, the capacity will decrease gradually since the CSI becomes less reliable. Moreover, the results show that the system can tolerate the estimation error variance up to about 0.01 with a slight degradation in the capacity. When the estimation error variance increases beyond 0.01 , however, the capacity performance will degrade notably. Besides, we may see that the capacity performance with $M=9$ in Fig. 3 is superior to that with $M=7$ in Fig. 2 due to great spatial diversity.

In Fig. 4, we plot the theoretical outage capacity and corresponding simulation of DAS with different receive antennas and estimation errors, where the outage probability $\varepsilon$ is set as 0.1 . $\sigma_{e}^{2}=0,0.3, \alpha=2$, and $N=1,2$. The accurate and approximate outage capacities are, respectively, calculated by (33) and (38), and they are referred as 'Theory 1' and 'Theory 2', respectively. As shown in Fig. 4, the theoretical values are all close to the corresponding simulated ones. Especially, the 'Theory 1' from the Newton's method has more accuracy than the 'Theory 2' from Gaussian distribution approximation, but the latter does not need iterative calculation. Due to the estimation error, the outage capacity of DAS with imperfect CSI $\left(\sigma_{\mathrm{e}}{ }^{2}=0.3\right)$ is lower than that with perfect CSI $\left(\sigma_{\mathrm{e}}{ }^{2}=0\right)$. Besides, the outage capacity of DAS with $N=2$ is higher than that with $N=1$ because multiple receive antennas are employed and more diversity is obtained. By comparing Fig. 2 and Fig. 4, it is observed that the outage capacity in Fig. 4 is lower than the corresponding ergodic capacity in Fig. 2 due to the constraint of outage probability.

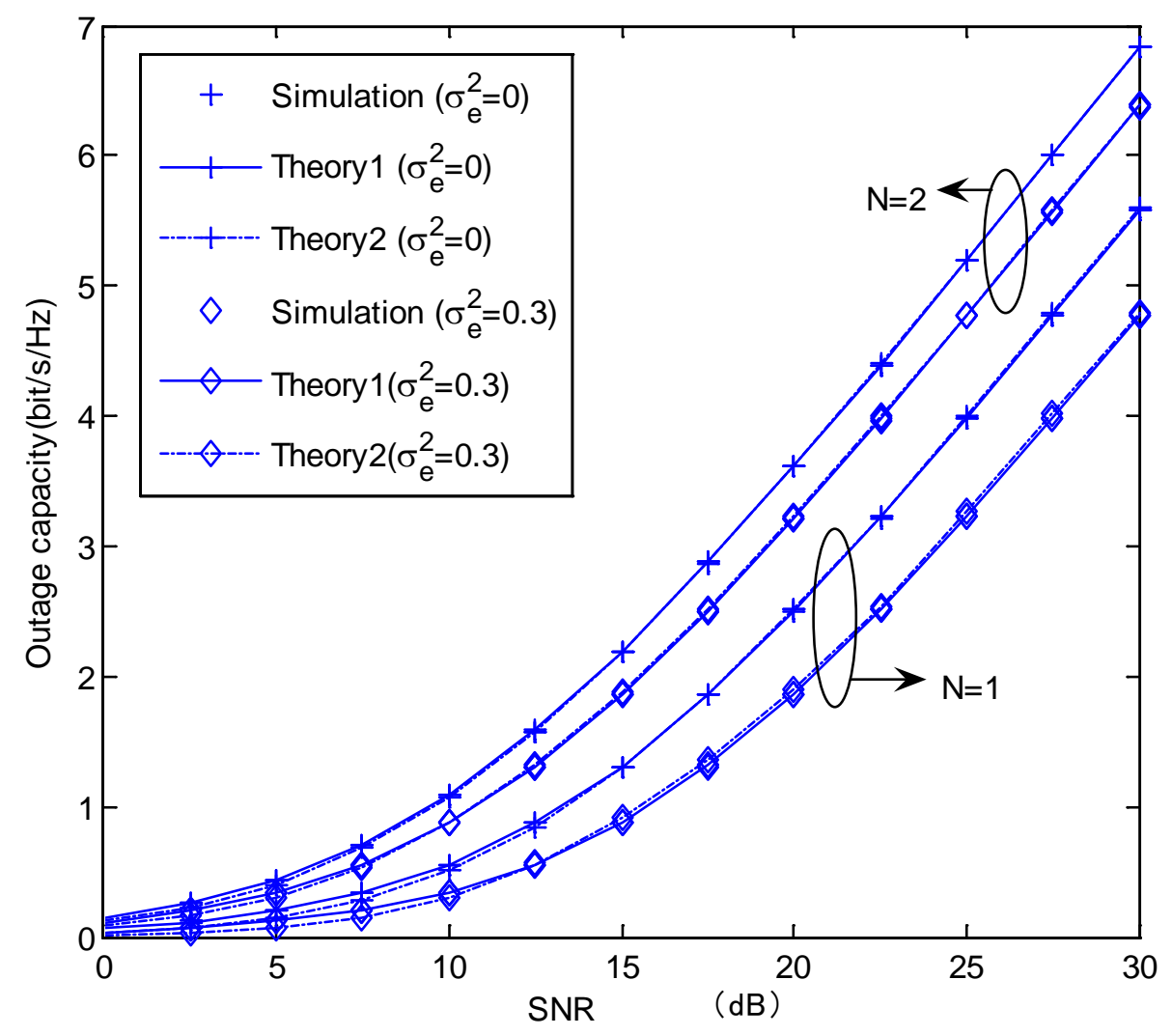

Fig. 4. Outage capacity of DAS different receive antennas and estimation errors. 
Fig. 5 shows the outage capacity of DAS with different path loss exponents and outage probability under imperfect CSI, where $N=2, \varepsilon=0.1,0.2, \sigma_{e}{ }^{2}=0.3$, and $\alpha=2$, 3. 'Theory 1 ' and 'Theory 2' are used to compute accurate and approximate theoretical outage capacity, respectively. From Fig. 5, we can see that the derived theoretical outage capacity matches the corresponding simulation result well, with only minor differences for 'Theory 2'. It is shown that the outage capacity of DAS with $\alpha=3$ is lower than that with $\alpha=2$ since the former experiences larger path loss. Besides, the outage capacity of DAS with $\varepsilon=0.2$ is higher than that with $\varepsilon=0.1$ since larger outage probability is allowed, which accords with the theoretical analysis in section 4 . The above results indicate that the derived theoretical formulae are valid and reasonable.

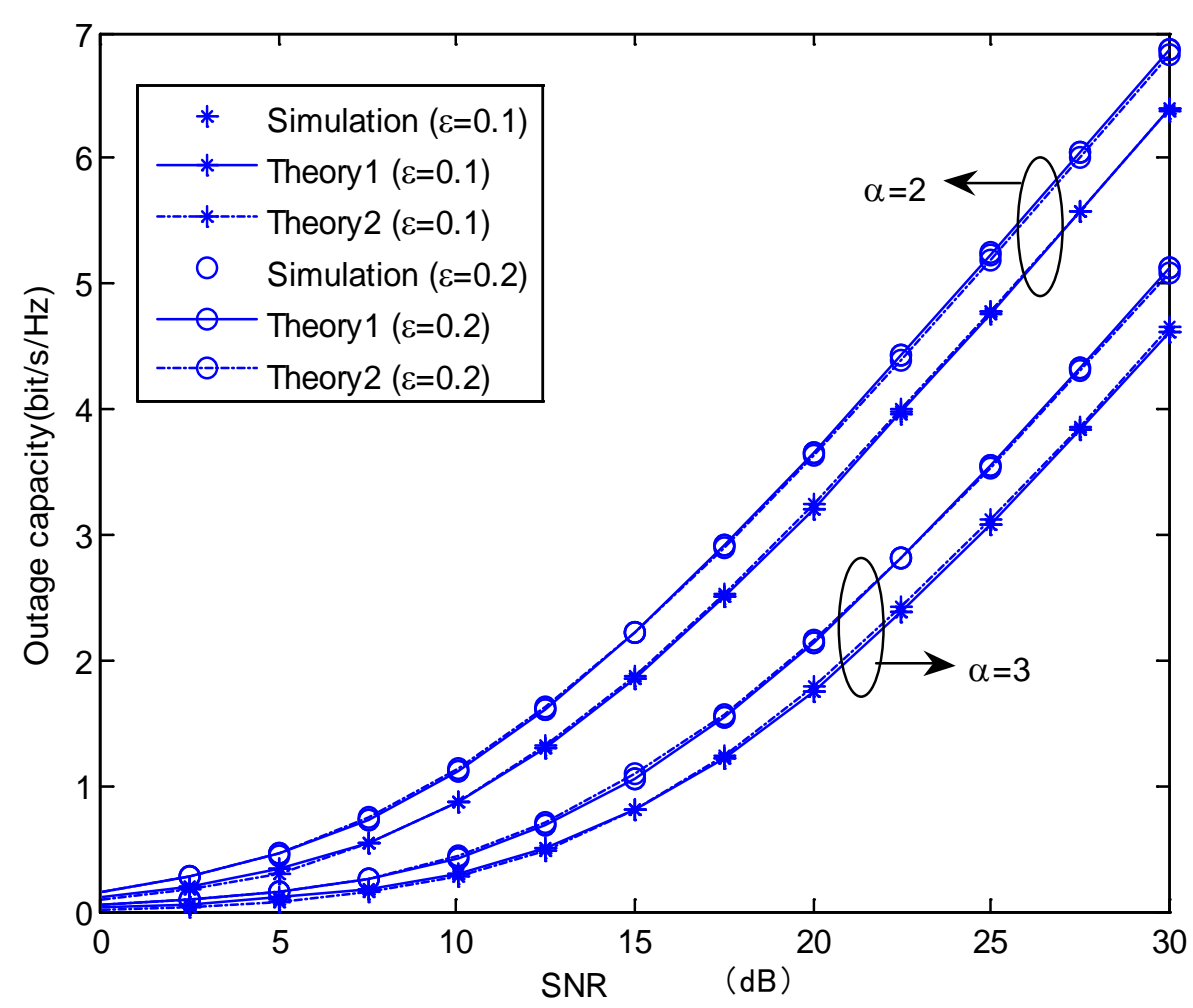

Fig. 5. Outage capacity of DAS different path loss exponents and outage probability.

In Fig. 6, we plot the theoretical outage probability and corresponding simulation of DAS with different receive antennas and estimation errors, where the outage capacity $C_{0}$ is set as to $2 \mathrm{bit} / \mathrm{s} / \mathrm{Hz}, \sigma_{\mathrm{e}}{ }^{2}=0,0.3, N=1,2$, and $\alpha=2$. The theoretical outage probability is calculated by (26). As shown in Fig. 6, the theoretical outage probability agrees the corresponding simulation well. It is shown that the outage probability of DAS with imperfect CSI $\left(\sigma_{\mathrm{e}}{ }^{2}=0.3\right)$ is higher than that with perfect CSI $\left(\sigma_{\mathrm{e}}{ }^{2}=0\right)$ due to the impact of estimation error. Besides, the system with two receive antennas has lower outage probability than that with single receive antenna since the former has greater diversity than the latter. The above results indicate that the derived outage probability is effective for the outage performance evaluation. 
Fig. 7 shows the outage probability of DAS with different path loss exponents and outage capacity constraints under imperfect CSI, where $N=2, \sigma_{\mathrm{e}}{ }^{2}=0.3, \alpha=2,3, C_{0}=2,4 \mathrm{bit} / \mathrm{s} / \mathrm{Hz}$. It is observed that the theoretical outage probability is still good agreement with the corresponding simulated one. Moreover, DAS with $\alpha=2$ has better outage performance than that with $\alpha=3$ because of smaller path loss, which accords with the existing knowledge. Besides, the outage probability with $C_{0}=2 \mathrm{bit} / \mathrm{s} / \mathrm{Hz}$ constraint is lower than that with $C_{0}=4 \mathrm{bit} / \mathrm{s} / \mathrm{Hz}$ constraint since smaller outage capacity is required. The above results further verify that the derived outage probability is valid and reasonable.

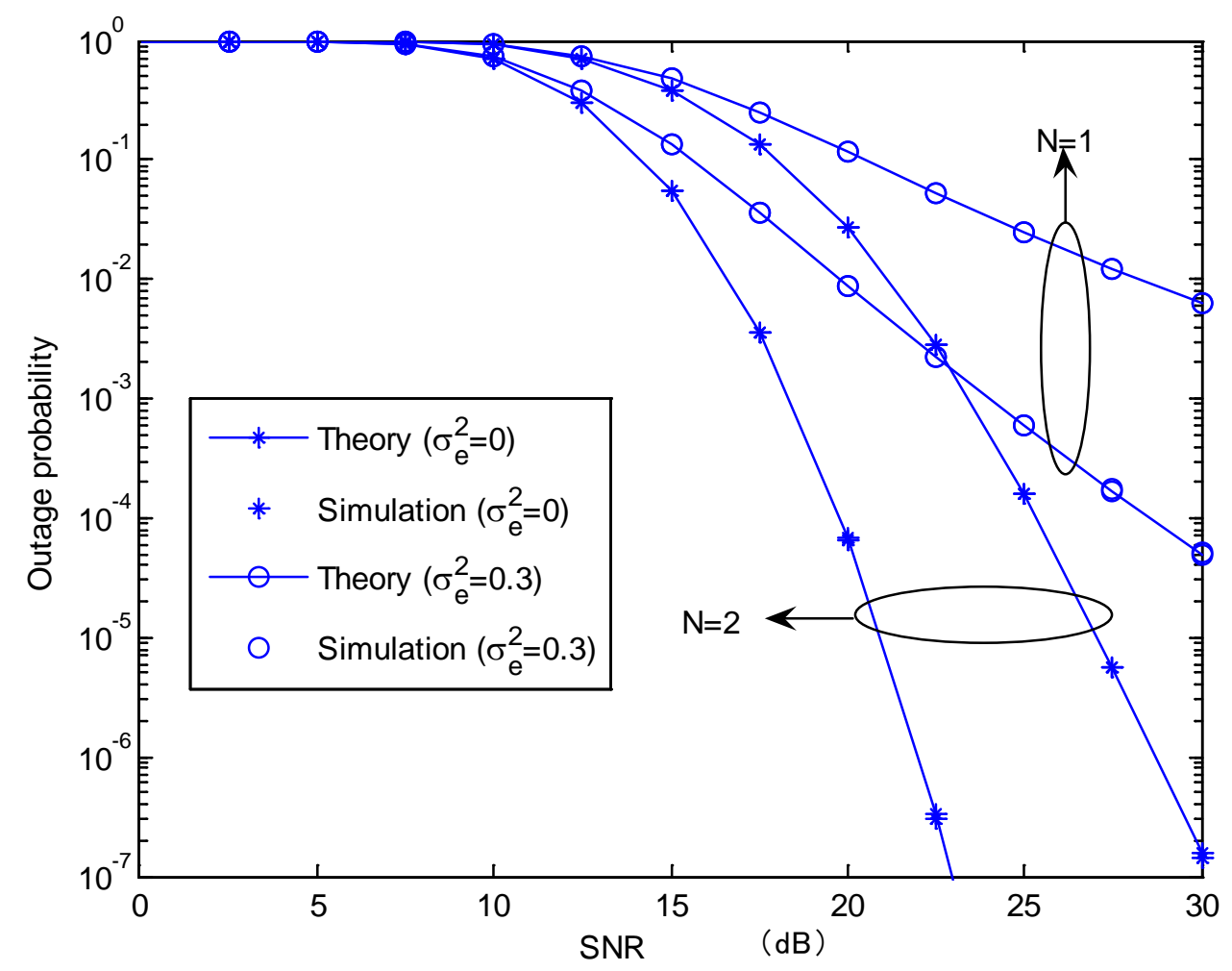

Fig. 6. Outage probability of DAS with different receive antennas and estimation errors.

\section{Conclusion}

We have studied the downlink performance of DAS with transmit antenna selection and multiple receive antennas in the presence of imperfect CSI, and analyzed the ergodic capacity and outage capacity over composite Rayleigh channel. The PDF of the effective output SNR is derived mathematically in detail. With this result, accurate closed-form expressions of ergodic capacity and outage probability of DAS are obtained, respectively. These expressions are in good agreement with the corresponding simulations, and include the ones under perfect CSI as special cases. For a given outage probability, a practical iterative algorithm based on Newton's method is presented to obtain the accurate outage capacity. Moreover, to avoid the iterative calculation of accurate outage capacity, an approximate closed-form outage capacity is also derived by using the Gaussian distribution approximation, and this approximate expression can simplify the calculation of accurate one due to its closed form. The analysis shows that the capacity is insensitive to the estimation error variance smaller than 0.01. Besides, simulation 
results verify the effectiveness of the theoretical analysis, and the system capacity can be improved effectively as the number of receive antennas increases and/or path loss exponent decreases and/or estimation error decreases. Based on this, these expressions may provide good performance evaluation for the DAS under imperfect CSI in theory, and avoid the time-consumed Monte Carlo simulation. Considering only downlink performance is analyzed in this paper, in the future work, we will further study the performance of uplink DAS with imperfect CSI, where transmit beamforming and receive combining vector will be considered. It is expected that superior performance can be achieved.

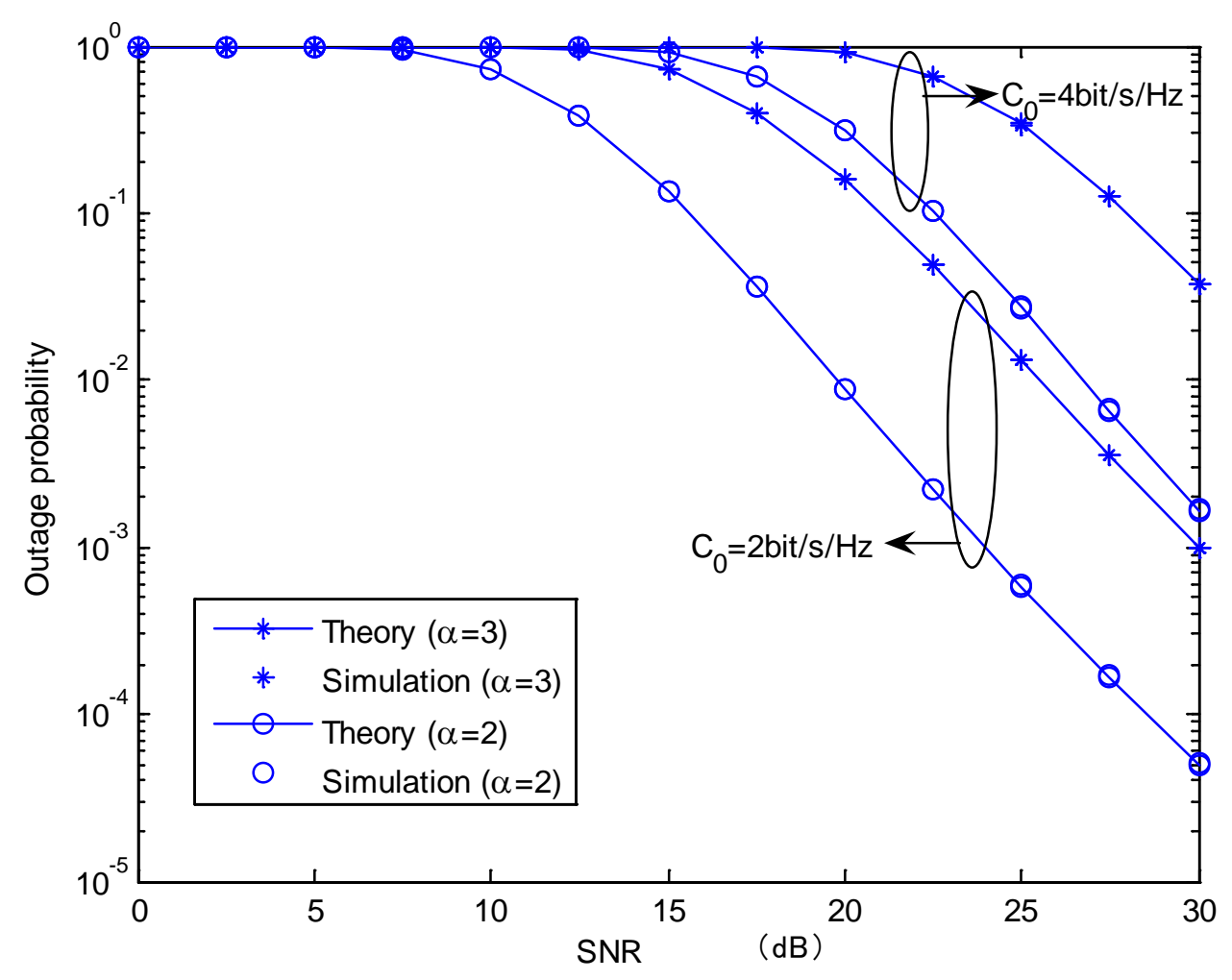

Fig. 7. Outage probability of DAS with different path loss exponents and outage capacities

\section{Appendix}

In this appendix, we will give the derivation of (20). Eq.(20) can be rewritten as:

$$
J=\int_{0}^{\infty} \rho^{t+N-1} \ln (1+\rho) e^{-\rho \lambda_{i}} d \rho=\frac{\Gamma(t+N)}{\lambda_{i}^{t+N}} \sum_{j=0}^{N+t-1} \int_{0}^{\infty} \frac{\lambda_{i}^{j} \rho^{j} e^{-\rho \lambda_{i}}}{j !(1+\rho)} d \rho
$$

which utilizes the equality $\rho^{n-1} e^{-\lambda_{i} \rho}=\left[\lambda_{i}^{-n} \Gamma(n)\right] \frac{d}{d \rho}\left(-\sum_{j=0}^{n-1}\left(\lambda_{i} \rho\right)^{j} e^{-\lambda_{i} \rho} / j !\right)$.

Let $x=1+\rho$, Eq. (39) can be changed to 


$$
\begin{aligned}
J & =\frac{\Gamma(t+N)}{\lambda_{i}^{t+N}} \sum_{j=0}^{N+t-1} \frac{\left(-\lambda_{i}\right)^{j}}{e^{-\lambda_{i}} j !} \int_{1}^{\infty} \frac{(1-x)^{j}}{x e^{\lambda_{i} x}} d x=\frac{\Gamma(t+N)}{\lambda_{i}^{t+N} e^{-\lambda_{i}}} \sum_{j=0}^{N+t-1} \frac{\left(-\lambda_{i}\right)^{j}}{j !} \sum_{k=0}^{j}(-1)^{k}\left(\begin{array}{l}
j \\
k
\end{array}\right) \int_{1}^{\infty} \frac{x^{k-1}}{e^{\lambda_{i} x}} d x \\
& =\frac{\Gamma(t+N) e^{\lambda_{i}}}{\lambda_{i}^{t+N}} \sum_{j=0}^{N+t-1} \frac{\left(-\lambda_{i}\right)^{j}}{j !} \sum_{k=0}^{j}(-1)^{k}\left(\begin{array}{l}
j \\
k
\end{array}\right) \lambda_{i}^{-k} \Gamma\left(k, \lambda_{i}\right)
\end{aligned}
$$

where $\Gamma(a, v) \triangleq \int_{v}^{+\infty} t^{a-1} e^{-t} d t$ is the upper incomplete Gamma function [31], and can be expressed as $\Gamma(u, v)=\Gamma(u) P_{u}(v)$ and $\Gamma(0, v)=E_{1}(v)$, where $\mathcal{P}_{u}(x)=\sum_{i=0}^{u-1} x^{i} e^{-x} / i$ ! is the Poisson distribution, and $E_{1}(\cdot)$ is the first order exponential integral function. Based on this, (40) can be simplified as

$$
J=\frac{\Gamma(t+N)}{\lambda_{i}^{t+N}}\left[\sum_{j=0}^{N+t-1} \frac{\left(-\lambda_{i}\right)^{j} e^{\lambda_{i}}}{j !} \Gamma\left(0, \lambda_{i}\right)+\sum_{j=1}^{N+t-1} \frac{\left(-\lambda_{i}\right)^{j} e^{\lambda_{i}}}{j !} \sum_{k=1}^{j}\left(-\lambda_{i}\right)^{-k}\left(\begin{array}{l}
j \\
k
\end{array}\right) \Gamma\left(k, \lambda_{i}\right)\right]
$$

Using Poisson distribution and $E_{1}(\cdot)$ function, and let $s=j-k$, (41) can be further simplified as

$$
\begin{aligned}
J & =\Gamma(t+N) \lambda_{i}^{-(t+N)}\left[\mathcal{P}_{N+t}\left(-\lambda_{i}\right) E_{1}\left(\lambda_{i}\right)+\sum_{k=1}^{N+t-1} \mathcal{P}_{k}\left(\lambda_{i}\right) \sum_{s=0}^{N+t-k-1}\left(-\lambda_{i}\right)^{s} e^{\lambda_{i}} /(s ! k)\right] \\
& =\Gamma(t+N) \lambda_{i}^{-(t+N)}\left[\mathcal{P}_{N+t}\left(-\lambda_{i}\right) E_{1}\left(\lambda_{i}\right)+\sum_{k=1}^{N+t-1} \mathcal{P}_{N+t-k}\left(-\lambda_{i}\right) \mathcal{P}_{k}\left(\lambda_{i}\right) / k\right]
\end{aligned}
$$

\section{Acknowledgment}

The authors would like to thank two anonymous reviewers for their valuable comments and excellent suggestions which considerably improved the paper.

\section{References}

[1] R. Heath, S. Peters, Y. Wang, J. Zhang, “A current perspective on distributed antenna systems for the downlink of cellular systems,” IEEE Communications Magazine, vol.51, no.4, pp. 161-167, 2013. Article (CrossRef Link)

[2] C.-X. Wang, X. Hong, X. Ge, X. Cheng, G. Zhang, J.S. Thompson, “Cooperative MIMO channel models: A survey,” IEEE Communications Magazine, vol.48, no.2, pp. 80-87, 2010. Article (CrossRef Link)

[3] Y. Lin,W. Yu, "Downlink spectral efficiency of distributed antenna systems under a stochastic model,” IEEE Transactions on Wireless Communications, vol.13, no.12, pp. 6891-6902, 2014. Article (CrossRef Link)

[4] X.You, D. Wang, B. Sheng, X. Gao, X. Zhao, M. Chen, "Cooperative distributed antenna systems for moblie communications," IEEE Wireless Communications, vol.17, no.3, pp. 35-43, 2010. Article (CrossRef Link)

[5] S. Zhou, M. Zhao, X.Xu, J. Wang, Y.Yao, "Distributed wireless communication systems: a new architecture for future public wireless access,” IEEE Communications Magazine, vol.41, no.3, pp. 108-113, 2003. Article (CrossRef Link)

[6] S. Sanayei, A. Nosratinia, “Antenna selection in MIMO systems,” IEEE Communication Magazine, vol.42, pp. 68-73, 2004. Article (CrossRef Link)

[7] Z.Chen, J.Yuan, B.Vucetic, "Analysis of transmit antenna selection/maximal-ratio combing in Rayleigh fading channel,” IEEE Transactions on Vehicular Technology, vol.54, no.4, pp. 1312-1321, 2005. Article (CrossRef Link) 
[8] W. Choi, J. D. Andrews, "Downlink performance and capacity of distributed antenna systems in a multicell environment,” IEEE Transactions on Wireless Communications, vol.6, pp. 69-73, 2007. Article (CrossRef Link)

[9] Y. Liu, J. Liu, H. Chen, et al., "Downlink performance analysis of distributed antenna systems," in Proc. of IEEE Wireless Communications and Signal Processing, pp.1-5, 2011. Article (CrossRef Link)

[10] X. Yu, Y. Yang, M. Li, M. Chen, "Capacity analysis of distributed antenna systems in MIMO Nakagami fading multicell environment,” Annals of Telecommunications, vol.67, pp.589-595, 2012. Article (CrossRef Link)

[11] J. Park, E. Song, W. Sung, "Capacity analysis for distributed antenna systems using cooperative transmission schemes in fading channels,” IEEE Transactions on Wireless Communications, vol.8, pp. 586-592, 2009. Article (CrossRef Link)

[12] L.Dai, "An uplink capacity analysis of the distributed antenna system (DAS): from cellular DAS to DAS with virtual cells,” IEEE Transactions on Wireless Communications, vol.13, no.5, pp. 2717-2731, 2014. Article (CrossRef Link)

[13] J. Wang, J. Wang, X. Dang, M. Lin, Y. Jiao, "System capacity analysis of downlink distributed antenna systems over composite channels," in Proc. of IEEE International Conference on Communication Technology, pp.1076-1079, 2010. Article (CrossRef Link)

[14] H. Chen and M. Chen, "Capacity of the distributed antenna systems over shadowed fading channels,” in Proc. of the IEEE Vehicular Technology Conference, pp. 1-4, 2009. Article (CrossRef Link)

[15] Y.Wen, J.Wang, M. Chen, "Ergodic capacity of distributed antenna systems over shadowed Nakagami-m fading channels," in Proc. of IEEE Wireless Communications and Signal Processing, pp.1-6, 2013. Article (CrossRef Link)

[16] J.-B. Wang, J.-Y. Wang, M. Chen, "Downlink system capacity analysis in distributed antenna systems,” Wireless Personal Communications, vol. 67, pp. 631-645, 2012. Article (CrossRef Link)

[17] W. Xu, Q. Wang, Y. Wang, B. Wu, "Downlink performance of distributed antenna systems in MIMO composite fading channel," KSII Transactions on Internet and Information Systems, vol.8, no.10, pp.3342-33603, 2014. Article (CrossRef Link)

[18] S.-R. Lee, S.-H. Moon, J.-S. Kim, I. Lee, "Capacity analysis of distributed antenna systems in a composite fading channel," IEEE Transactions on Wireless Communications, vol. 11, no. 3, pp.1076-1086, 2012. Article (CrossRef Link)

[19] M. Matthaiou, N.D. Chatzidiamantis, G.K.Karagiannidis, "A new lower bound on the ergodic capacity of distributed MIMO systems,” IEEE Signal Processing Letters, vol.8, pp.227-230, 2011. Article (CrossRef Link)

[20] H. Chen, J.Wang, M. Chen, “Outage capacity study of the distributed MIMO system with antenna cooperation,” Wireless Personal Communications, vol. 59, no.4, pp.599-605, 2011. Article (CrossRef Link)

[21] W. Roh and A. Paulraj, "Outage performance of the distributed antenna systems in a composite fading channel," in Proc. of IEEE Vehicular Technology Conference, vol.3, pp. 1520-1524, 2002. Article (CrossRef Link)

[22] J.Wang, J.Wang, M. Chen, H. Chen, X. Dang, "System outage probability analysis of uplink distributed antenna systems over a composite channel," in Proc. of IEEE $73^{\text {rd }}$ Vehicular Technology Conference, pp. 1-5, 2011. Article (CrossRef Link)

[23] H. Chen, J. Wang, M. Chen, "Outage performance of distributed antenna systems over shadowed Nakagami-m fading channels,” European Transactions on Telecommunicatons, vol.20, pp. 531-535, 2009. Article (CrossRef Link)

[24] J.Wang, M.Chen, Q.-S. Hu, "System outage probability analysis of distributed antenna systems over shadowed generalized-gamma composite channels," in Proc. of IEEE Wireless Communications and Signal Processing, pp.1-6, 2012. Article (CrossRef Link)

[25] F. Héliot, R. Hoshyar, R. Tafazolli, "A closed-form approximation of the outage probability for distributed MIMO systems," in Proc. of IEEE 10th Workshop on Signal Processing Advances in Wireless Communications, pp.529-533, 2009. Article (CrossRef Link) 
[26] L. Dai, S. Zhou, Y. Yao, "Capacity analysis in CDMA distributed antenna systems," IEEE Transactions on Wireless Communications, vol.4, pp.2613-2620, 2005. Article (CrossRef Link)

[27] Z. Shi, H. Leib, "Transmit antenna selected V-BLAST systems with power allocation," IEEE Transaction on Vehicular Technology, vol.57, no.4, pp. 2293-2304, 2008. Article (CrossRef Link)

[28] Q. Kuang, S.-H.Leung, X.Yu, "Adaptive modulation and joint temporal spatial power allocation for OSTBC MIMO systems with imperfect CSI," IEEE Transaction on Communications, vol.60, no.7, pp. 1914-1924, 2012. Article (CrossRef Link)

[29] M.K.Steven, Fundamentals of statistical signal processing: estimation theory, Prentice Hall, 1998.

[30] J. G. Proakis, Digital communications, 4th ed, McGraw-Hill, New York, 2001.

[31] I. S. Gradshteyn and I. M. Ryzhik, Table of integrals, series, and products, 7th ed. San Diego, CA: Academic, 2007.

[32] J. Romero-Jerez and A. Goldsmith, "Performance of multichannel reception with transmit antenna selection in arbitrarily distributed Nagakami fading channels," IEEE Transactions on Wireless Communications, vol.8, no.4, pp. 2006-2013, 2009. Article (CrossRef Link)

[33] M.Torabi, J.F.Frigon, S.Brunilde, "Performance analysis of adaptive modulation in multiuser selection diversity systems with OSTBC over time-varying channels," IEEE Signal Processing Letters, vol.19, no.4, pp. 211-214, 2012. Article (CrossRef Link)

[34] E. Biglieri, R.Calderbank, A. Constantinides, et. al., MIMO wireless communications. Cambridge, U.K.: Cambridge Univ. Press, 2007. Article (CrossRef Link)

[35] E.G.Larsson, P.Stoica, Space-time block coding for wireless communications. Cambridge, U.K.: Cambridge Univ. Press, 2003. Article (CrossRef Link)
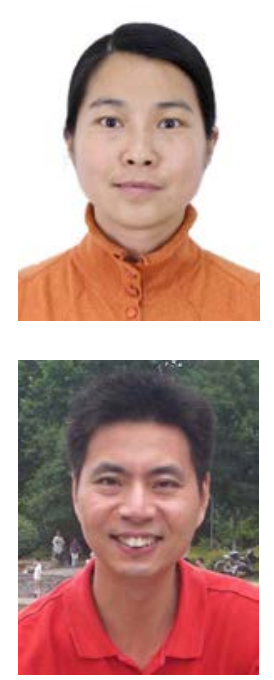

Weiye Xu received her M.S. degree in Communication and Information Systems from Hohai University. She is pursuing her Ph.D. at PLA University of Science and Technology, China. She is an associate professor at the School of Communication Engineering at Nanjing Institute of Technology. Her research interests include digital communication, MIMO technique, and distributed antenna systems.

Min Lin received his Ph.D. in Electrical Engineering from Southeast University, Nanjing, in 2008. He is currently an adjunct professor at PLA University of Science and Technology, Nanjing. He is the author and co-author of more than 80 papers and holds four patents. His research interests include wireless communications and array signal processing. 